\title{
Evaluating the safety and immunogenicity of yellow fever vaccines: a systematic review
}

\author{
This article was published in the following Dove Press journal: \\ Vaccine: Development and Therapy \\ 20 April 2015 \\ Number of times this article has been viewed
}

\section{Roger E Thomas}

Department of Family Medicine, G0I2 Health Sciences Center, University of Calgary Medical School, Calgary, AB, Canada
Correspondence: Roger E Thomas Department of Family Medicine, GOI2 Health Sciences Center, University of Calgary Medical School, 3330 Hospital Drive NW, Calgary, AB T2N 4NI, Canada

Tel +l 4032109208

Fax +I 4032704329

Email rthomas@ucalgary.ca
Purpose: To review the safety and immunogenicity of yellow fever vaccines.

Literature search: The Cochrane Library (including the Cochrane CENTRAL Register of Controlled Trials, the Cochrane Database of Systematic Reviews, and the NHS Database of Abstracts of Reviews of Effects); MEDLINE; EMBASE; BIOSIS Previews; Global Health; CAB Abstracts; and the Lilacs Database of Latin American and Caribbean literature were searched for individual studies and systematic reviews through January 1, 2015.

Results: Six yellow fever vaccines are currently produced, and they are effective against all seven yellow fever virus strains. There is a $99.2 \%$ homology of the genome sequences of the six current vaccines. Four systematic reviews identified very small numbers of serious adverse events. A systematic review (updated) of all published cases identified 133 serious adverse events that met the Brighton Collaboration criteria: 32 anaphylactic, 42 neurologic (one death), 57 viscerotropic (25 deaths), and two of both neurologic and viscerotropic SAEs. The Sanofi Pasteur Global Pharmacovigilance database reported 276 million doses of Stamaril ${ }^{\text {TM }}$ distributed worldwide and identified 12 reports of yellow fever vaccine-associated viscerotropic disease (YEL-AVD), 24 of yellow fever vaccine-associated neurologic disease (YEL-AND), and 33 reports of anaphylaxis (many already published). The Biomanguinhos manufacturer's database reported 110 million doses distributed worldwide between 1999 and 2009, and the rate of YEL-AND was estimated at 0.084/100,000 doses distributed and YEL-AVD at 0.02/100,000 doses distributed.

Conclusion: Reports of serious adverse events are mostly from travelers from developed countries, and there is likely serious underreporting for developing countries. On the basis of the published reports, the yellow fever vaccines are very safe and probably provide life-long immunity.

Keywords: yellow fever vaccine, safety, serious adverse events, vaccine genome homology, immunogenicity, systematic reviews

\section{Introduction}

Yellow fever (YF) is caused by the yellow fever virus (YFV) transmitted by mosquitoes in 31 countries in sub-Saharan Africa and 13 in Central and South America. ${ }^{1}$ The virus is a single strand RNA virus, 10,800 nucleotides in length; the C protein surrounds the genome, the $\mathrm{M}$ and $\mathrm{E}$ proteins are on the outside of the virion, and the $\mathrm{E}$ protein is the major immunogen. ${ }^{2}$ The WHO estimated from the early 1990 s onward that annually there were 200,000 new cases of YF and 30,000 deaths worldwide, with $90 \%$ in Africa, with the 2013 update being 84,000-170,000 severe cases and 29,000-60,000 deaths annually in Africa alone. ${ }^{1}$ Many cases in developing countries are likely not to be reported; travelers from developed countries may have more resources to limit 
exposure to mosquitoes, and their cases have a higher probability of being recognized and published. Key problems are definitively verifying the cases by accepted criteria and extensive laboratory testing, and identifying denominators of vaccinated individuals. Most reports of adverse events (AEs) and serious adverse events (SAEs) are about travelers, and $95 \%$ of reports are from countries where only $5 \%$ of doses are distributed. ${ }^{1}$

There are currently six manufacturers of the $17 \mathrm{D}$ vaccine producing 30-60 million doses annually. Although the seven wild-type YFV genotypes differ by up to $25.1 \%$ at the nucleotide and $7.9 \%$ at the amino acid level, 17D vaccines are effective against all seven. ${ }^{2}$ All vaccines are based on a wild-type virus isolated in 1927 from an individual in Ghana named Asibi. The three main vaccine substrains are YF17D-204, YF17D-203, and YF17DD, and the method of production in chicken embryos has remained unchanged for the past 25 years. Lot potency is measured by plaque assay, with a minimum required potency of $10^{3}$ mouse $\mathrm{LD}_{50}$ per dose or equivalent in plaque forming units. ${ }^{2}$

The data on the phylogeny of the YF vaccine based on passaging has recently been updated ${ }^{3}$ by viral sequencing of these strains: RKI YFV vaccine, Crucell Switzerland AG (strain 17D-204, substrain 112/95, passage 238) (Accession number: JN628279); Flavimun working seed lot (WSL), Crucell Switzerland AG (strain 17D-204, substrain 112/95, passage 237) (Accession number: JN628280); TVX Flavimun vaccine, Crucell Switzerland AG (origin 17D-204, substrain 112/95, passage 238) (Accession number: JN628281); and Stamaril $^{\circledR}$ Yellow fever live vaccine, Sanofi Pasteur MSD (Ch.-Nr.: Z6329-2). ${ }^{3}$ Stock et $\mathrm{al}^{3}$ also obtained full genome sequences from the EMBL GenBank for YFV-17DD Brazil (DQ100292), YF-AVD2791-93F 04 from Spain (DQ118157), YFV 17D-Tiantan, People's Republic of China (FJ654700.1), YFV-17DD case number 1 YF vaccine-associated viscerotropic disease (YEL-AVD) Peru (GQ379162.1), YFV-17DD case number 2 YEL-AVD Peru (GQ379163.1), YFV-17DD (U17066), YFV-17D-213 (U17067), YFV French neurotropic strain (U21055), YFV-17D-204 1 (USA) (X03700), YFV17D-204 2 (Pasteur) (X15062), YFV French viscerotropic strain (U21056), YFV Asibi (AY640589), and Dengue 1 (NC 001477).

For these strains, there is an overall homology of $99.2 \%$, with 84 sequence differences in the entire genome, of which 29 have an effect on protein levels and accumulate within the range of the E protein, thus indicating the genetic stability of these YF vaccine strains. The 17-DD used in South America has 56 nucleotide differences (of which 18 affect protein levels) from the 17D strains. Only 12 of these nucleotide differences are common to all four 17-DD strains. ${ }^{3}$

Within the 17-D group there are three clades: the Crucell, WHO 17D-213, and the former RKI (Robert Koch Institute, Berlin) strains; the US and Pasteur strains form a second clade; and the Stamaril strain (derived from an YEL-AVD case in Spain after vaccination with Stamaril ${ }^{\text {TM}}$ ) and the Chinese strain (which separated from the others at passage 229 with six amino acid changes) form a third clade. ${ }^{3}$ The overall $99.2 \%$ homology shows the vaccines are remarkably similar, and Stock et $\mathrm{al}^{3}$ propose that monitoring genome sequences would provide a simpler method of monitoring vaccine batch safety than intracerebral injection into monkeys and detailed subsequent histological examinations. The derivation of the current strains ${ }^{4}$ and their genome nucleotide sequences $^{3}$ are available in flow charts.

The immune response to YFV is complex. IgM antibodies peak by 2 weeks after vaccination, then decline over the next few months. Neutralizing antibodies, the key method of protection, are apparent by the 7th day. Ninety-eight percent of vaccinees are fully protected by a neutralization titer of $1: 10$ for $\geq 10$ years, and the antibodies probably persist lifelong. There is strong induction of CD4+ (day 7) and CD8+ (day 30) cytotoxic T-cell responses against the E structural and the nonstructural NS1, NS2B, and NS3 proteins. The vaccine also replicates in dendritic cells and stimulates proinflammatory cytokines. $^{2}$

A study of the T-cell response of nine individuals vaccinated with $17 \mathrm{D}$ used a library of 851 peptides spanning the entire F-17D polyprotein and organized into 60 pools to map T-cell epitopes. There was a strong CD8+ T-cell response targeting several epitopes within each viral protein, and the YF-17D-specific memory CD8+ T-cells subsequently showed robust proliferative potential memory and were polyfunctional in terms of degranulation and production of multiple cytokines. Memory cells could be rapidly recruited to prevent subsequent infections. ${ }^{5} \mathrm{~A}$ study of the T-cell responses of 21 individuals who received the 17D-204 vaccine using 78 predicted epitopes similarly noted the CD8+ response matured from an effector- to a memory-type response. ${ }^{6}$

In a study comparing responses of younger and older individuals, the power computation based on expected $80 \%$ virus neutralization of $95 \%$ in the younger and $66 \%$ in the older group with $\alpha=0.05$ and $\beta=0.2$, estimated that 26 participants per group were needed. The study compared 30 healthy individuals $18-28$ years of age and 28 individuals 60-81 years of age and found that 10 days after vaccination with 17D Stamaril ${ }^{\mathrm{TM}}$ (Lot no B5355, Sanofi Pasteur, France) 
seroprotection of $80 \%$ of the virus by neutralizing antibodies was obtained by $77 \%$ of the young and $50 \%$ of the older group and by $100 \%$ of both by day 30 ; viremia was noted in $53 \%$ of the younger and $82 \%$ of the older group at day 5 and in $0 \%$ of both groups at day 14 . The authors postulated that a weaker immune response allowed higher viremia levels in the older group and might expose them to more SAEs. ${ }^{7}$

\section{Overview of reported adverse events and history of use}

AEs following immunization (AEFI) are classified by the WHO as serious if they result in persistent or significant disability, a congenital anomaly or birth defect, intervention to prevent permanent impairment, hospitalization or prolongation of hospitalization, or death. The intensity of adverse events is classified as mild, moderate or severe. ${ }^{3}$

The WHO classifies SAEs following immunization (AEFI) with YF vaccine into three categories: immediate severe hypersensitivity or anaphylactic reactions; YF vaccine-associated neurologic disease (YEL-AND) resulting in meningitis, encephalitis, or an autoimmune reaction (Guillain-Barré syndrome), or acute disseminated encephalomyelitis (ADEM); orYEL-AVD causing multiorgan system dysfunction or failure. ${ }^{1}$ YEL-AVD was first recognized in 2001, and cases before that would need to be recognized and assessed by the multiorgan dysfunction involved.

SAEs occur only in first-time YF vaccinees, and no nucleotide mutations have been found in vaccine viruses isolated from individuals who experienced YF-AVD. ${ }^{2}$

\section{Systematic reviews}

There are four systematic reviews of SAEs in general populations. One assessed all published reports of SAE's. ${ }^{9}$ Three reviews assess databases of active and passive surveillance, ${ }^{10}$ reporting rates in pharmacovigilance databases, ${ }^{11}$ and groups usually estimated at high risk. ${ }^{12}$ These databases report on millions of vaccinees, but rarely provide enough data to assess with Brighton or CDC criteria.

\section{Published cases}

A systematic review of published cases ${ }^{9}$ assessed cases with the Brighton Collaboration YF SAEs criteria ${ }^{13-16}$ in a specially designed computerized Microsoft Access database. A new search on January 1, 2015 updated the database. ${ }^{17,18}$ There are 32 anaphylaxis cases, 42 neurologic cases (one death), 57 viscerotropic cases (25 deaths), and two cases with both neurologic and viscerotropic symptoms (Table 1). All SAEs occurred following the first YF vaccination.
An additional 345 cases were presented with insufficient detail to meet Brighton Collaboration criteria: 173 neurological, 68 viscerotropic (24 deaths), 67 anaphylaxis, and 34 cases from a UK database and 3 from a Swiss database described as "SAEs" but not further classified into neurologic or viscerotropic. Reasons why potential cases did not meet the Brighton Collaboration criteria include no medical or surgical history, minimal clinical and/or laboratory data, insufficient testing for YFV and YF vaccine virus, or no liver histopathology to meet Brighton viscerotropic disease criteria. Cases of potential anaphylaxis were not assessed for a history of allergies or reactions to eggs. In many African and Latin American countries where YF vaccine is administered, patients, especially in remote areas, may not have ready access to health care, or the system is not equipped to identify cases and use appropriate clinical histories and laboratory tests to rule in adverse reactions to YF vaccine and rule out other pathogens. ${ }^{11}$ Vaccination campaigns involving 38 million individuals in eight African countries required a huge amount of administrative work to ascertain adverse events and yet many tests were missing for many individuals. ${ }^{19}$ Passive surveillance systems may miss many SAEs and often do not go back to the source to check details, and thus there is unknown under-detection of cases.

The January 1, 2015 update also assessed vaccine strains in the published cases: 149 were identified only as 17D, 36 as 17D-204, and 15 as 17DD. In 28 publications, the strain was not stated: almost certainly the 26 from Europe and North America are 17D, and the two from South America are 17DD. If these are added to the articles with stated strains, the totals

Table I Published cases of serious adverse events (SAEs) following yellow fever vaccination

\begin{tabular}{ll}
\hline SAEs & Number of cases \\
\hline Meeting Brighton Collaboration criteria & \\
Anaphylactic & 32 \\
Neurologic & 42 (I death) \\
Viscerotropic & 57 (25 deaths) \\
Both neurologic and viscerotropic & 2 (also 2 proven \\
& due to wild virus) \\
Total & 133 \\
Not meeting Brighton Collaboration criteria & \\
Anaphylactic & 67 \\
Neurologic & 173 \\
Viscerotropic & 68 (24 deaths) \\
Not classified as neurologic or viscerotropic & 37 \\
Total & 345 \\
Insufficient data to be regarded as yellow & 254 \\
fever vaccine related-SAE & \\
\hline
\end{tabular}


are 175 17D (of which 110 met no Brighton Collaboration criteria), 36 17D-204, 17 17DD, and one unknown (People's Republic of China).

There was limited identification of strain and manufacturer: YF-VAX Connaught USA (5), YF-VAX SanofiPasteur USA (13), YF-VAX Sanofi-Pasteur (3), Stamaril (2), Stamaril Sanofi-Pasteur (13), Stamaril Aventis-Pasteur (4), Biomanguinhos (17), and one each for the South Africa National Institute for Virology Rockefeller Foundation, Merrill National, National Drug Co. Philadelphia, Wellcome, and the Indisch Institut Amsterdam.

A further 253 cases were excluded as presenting insufficient data to be regarded as YF vaccine-related SAEs. It is clear that assessing SAEs from individual published cases cannot provide an unbiased estimate.

\section{Active and passive surveillance}

The systematic review of YF vaccine-related SAEs ${ }^{10}$ identified 66 relevant studies of which 24 used active surveillance, 25 passive, and 17 both (15 are pharmacovigilance databases). The active surveillance databases included 2,660,929 patients from general populations, heavily weighted $(97.7 \%)$ by the large Brazilian study. There were no observed cases of viscerotropic or neurotropic disease, one of anaphylaxis, and 26 cases of urticaria (hypersensitivity). No SAEs were observed in four studies of infants and children $(n=2,199)$, four studies of women $(n=1,334)$, and one study of $174 \mathrm{HIV}+$ patients.

Six passive surveillance studies of campaigns in general populations included 94,500,528 individuals, very heavily weighted (99\%) by the Brazilian data, and resulted in an estimate of 0.51 serious AEFIs/million doses. Five retrospective reviews of hospital or clinic records (heavily weighted [96\%] by the data from the Hospital for Tropical Diseases, London) included 60,698 individuals: no serious AEFIs were identified. Two studies included 35,723 children, four studies included 138 pregnant women, six studies included $191 \mathrm{HIV+}$ patients, and a review of patients who were $\mathrm{HIV}+$, and no serious AEFIs were proven. The databases in each country used different definitions, protocols, and surveillance mechanisms for initial identification and reporting of cases, and strategies for the clinical and laboratory case follow-up.

The pharmacovigilance databases provide three sets of estimates. The Brazilian and Australian estimate is low, the US VAERS data medium, and the UK and Swiss data higher. The active surveillance data estimate is lower, and is strongly influenced by the Brazilian database. The review concluded that the ability to detect, assess, and report SAEs is strongly biased toward individuals from developed countries. Nevertheless the 17D and 17DD YF vaccine are very safe and highly effective against an illness with high potential mortality rates.

\section{Pharmacovigilance databases}

A systematic review of pharmacovigilance databases ${ }^{11}$ identified 15 pharmacovigilance databases. Ten contributed data for the review and the $107,621,154$ patients were heavily weighted $(94 \%)$ by the Brazilian database. The Australian estimates were low at $0 / 210,656$ for "severe neurological disease" and 1/210,656 for YEL-AVD, and also the Brazilian was low with nine hypersensitivity, 0.23 anaphylactic shock, 0.84 neurologic syndrome, and 0.19 viscerotropic events/million doses. The five analyses of the US VAERS database are for partly overlapping periods and estimate 6.6 YEL-AVD and YEL-AND cases per million, and 11.1-15.6 "SAEs" per million. The UK estimates of 34 "SAEs" and the Swiss with 14.6 "neurologic events" and 40 "serious events not neurological" per million doses were higher. The review concluded that reporting rates for SAEs following YF vaccination are low. Differences in reporting rates may be due to differences in definitions, surveillance system organization, methods of reporting cases, administration of YF vaccine with other vaccines, incomplete information about denominators, time intervals for reporting events, the degree of passive reporting, access to diagnostic resources, differences in time periods of reporting, and degree of follow-up for more data. ${ }^{11}$

\section{Manufacturer database reports on individual vaccines}

There are detailed reports ${ }^{20}$ about the Sanofi-Pasteur 17D-204 Stamaril ${ }^{\mathrm{TM}}$ and the Biomanguinhos 17DD vaccine. ${ }^{21}$

\section{Sanofi-Pasteur I7D-204 Stamaril ${ }^{\mathrm{TM}}$}

More than 300 million doses of the 17D-204 YF vaccine Stamaril $^{\text {TM }}$ by Sanofi-Pasteur have been distributed since 1983. The safety profile of the 276 million distributed since an electronic pharmacovigilance database was implemented in 1993 by Sanofi-Pasteur has been published..$^{20}$ The SanofiPasteur Global Pharmacovigilance department received 1,460 medically-confirmed reports from health care professionals, health authorities, and scientific publications from January 1, 1993 to September 30, 2010, of which 805 met the seriousness criteria. Cases of YEL-AND and YEL-AVD were assessed with the CDC YF Vaccine Working Group case definitions and the Brighton Collaboration YEL-AVD 
definition, and anaphylactic reactions with the MedDRA queries and the Brighton criteria. ${ }^{20}$

Twelve reports of YEL-AVD were identified (most already published as case reports) and classified by Brighton Collaboration criteria as two Definite, three Probable, two Suspect, and five insufficient data (two patients died and ten recovered). Four patients were reported to have received concomitant inactivated vaccines. The estimated rate for travelers based on these data is $0.07 / 100,000$ doses distributed and 0.004/100,000 doses worldwide. Twentyfour reports of YEL-AND were identified (six already published) with four Definite, six Probable, and 14 Suspect according to CDC YF Working Group criteria (one died, 15 recovered, and eight were lost to follow-up). Seven patients had received at least one other vaccine. The estimated rate for travelers from nonendemic countries based on these data is $0.15 / 100,000$ doses distributed and $0.008 / 100,000$ doses worldwide. There were 19 reports of immunosuppressed patients having received Stamaril ${ }^{\mathrm{TM}}$, but no cases among them of YEL-AND or YEL-AVD. There were 33 reports of anaphylaxis meeting the Brighton Collaboration level 1, 2, or 3 criteria (including 12 possible anaphylactic shock) for a rate of 0.2/100,000 doses distributed in Europe and $0.01 / 100,000$ doses worldwide. Seven individuals received concomitant vaccines. Inadvertent vaccination during pregnancy was reported 195 times with 109 fetal outcomes reported, and the assessment was that "Our review of these cases did not reveal any safety concern". The report also concluded that: "there is no evidence in our analysis that suggests that the safety profile of this live-attenuated vaccine in adults older than 60 years is less favourable than in younger adults". ${ }^{20}$ Nine clinical trials of Stamaril ${ }^{\mathrm{TM}}$ were also conducted with 1,900 individuals (including 626 children, $102 \mathrm{HIV}+$ adults, and 43 elderly individuals) and no SAEs were reported..$^{20}$ The immunogenicity of the Aventis-Pasteur Stamaril and Berna Flavimun 17D strains were compared in 304 healthy volunteers aged 18-60 years who had not previously received YF vaccine. Neutralizing antibody was defined as the reciprocal of the highest dilution of serum that neutralized at least $50 \%$ of viral plaques. Groups were well matched at baseline. Seven of the 151 in the Flavimun group and 4 of the 153 in the Stamaril group were seropositive at baseline and excluded. At 29 days after vaccination, the Geographic Mean Titer (GMT) of neutralizing antibodies was 1,184 in the Flavimun and 612 in the Stamaril group $(P<0.0001)$. Seroprotection at neutralizing titers $\geq 1: 10$ was $100 \%$ in both groups, at $\geq 1: 150$ was $95 \%$ in the Flavimun and $87.5 \%$ in the Stamaril group, and at $\geq 1: 450$ was $85 \%$ in the Stamaril group and $67 \%$ in the Flavimun group. In the Flavimun group the 29 day GMT for males was 1,465 and for females was $953(P=0.022)$, and for the Stamaril group an opposite pattern was noted, 502 for males and 709 for females $(P=0.229) .{ }^{4}$

Solicited reactions to vaccine were reported by $37 \%$ in both groups (headache, asthenia, myalgias, arthralgia, and gastrointestinal disorders) and unsolicited reactions by $21 \%$ of the Flavimun and $15 \%$ of the Stamaril recipients. Older recipients reported fewer side effects $(P=0.003)$.

The full consensus nucleotide sequence of 12 bulk lots of Stamaril vaccine from 1990 to 2002 was compared, and only four nucleotide differences were found, but no mutations. ${ }^{22}$ Flaviviruses lack proofreading enzymes for RNA replication and point mutations could accumulate; the constancy of the genome is thus remarkable. The distribution into smaller and a few larger plaques was also constant, potency values remained above the minimum, and total viral load as measured by RT-PCR was highly homogeneous, confirming the stability and safety of the vaccine. ${ }^{22}$ In 2010, Sanofi-Pasteur tested two new candidate WSLs at passage 233 to replace the YF17D-204 PV26/S706 (passage 234) that had been in continuous manufacturing use for 20 years and found no significant differences. ${ }^{23}$

\section{Biomanguinhos I7DD vaccine}

Approximately 110 million doses were distributed between 1999 and 2009 and the rate of YEL-AND was estimated at 0.084/100,000 doses distributed and YEL-AVD at $0.02 / 100,000$ doses distributed. ${ }^{21}$

\section{Risk factors and at-risk groups}

The literature usually identifies pregnant females, HIV+ individuals, immunosuppressed individuals, and the elderly as at-risk from YF vaccination. A systematic review of these groups identified the following studies of AEs; nine in infants and children, eight in pregnant women, nine in HIV+ patients, five in persons 60 years and older, one in individuals taking immunosuppressive medications, and two of maternalneonate transmission. No other SAEs were identified in the other studies of vulnerable groups. ${ }^{12}$

For persons $\geq 60$ years, the five passive surveillance databases identified very small numbers of cases of YFV-AVD, YFV-AND, and anaphylaxis. The unavailability of definite denominators made it inappropriate to compute rates. ${ }^{12}$

The review ${ }^{12}$ identified these problems in assessing SAEs in older individuals: The time periods of the three reports from the US VAERS pharmacovigilance database 
overlap. Khromava et $\mathrm{al}^{24}$ reviewed cases from 1990 to 2002 and identified 188,870 individuals aged 60-69 years and 93,565 individuals $\geq 70$ years. Lindsey et al, ${ }^{25}$ for the slightly overlapping period of 2000-2006, identified 191,025 individuals aged $60-69$ years and 87,177 individuals $\geq 70$ years. Martin et al, ${ }^{26}$ for $1990-1998$, noted that 1,443,686 doses of YFV were administered to civilians of all age groups and identified 19 cases of serious adverse reactions in which YFV alone was administered and 16 cases of serious adverse reactions in which it was administered with other vaccines. The estimated age distribution of travelers from GeoSentinel clinics receiving vaccine in 1998 was applied to the VAERS data for 1990-1998. For the 722 AEs in Khromava et al's study, ${ }^{24}$ only YF vaccine was administered in $26 \%$ of cases, other known vaccines were administered in $50 \%$ of cases, and the vaccine is not stated in $24 \%$. In Lindsey et al's study, ${ }^{25}$ AEs involved YF vaccine alone in 190 cases and other vaccines in 470 cases. The numbers of SAEs are small. In those aged 60-69 years in Khromava et al's study, ${ }^{24}$ there were eight SAEs (two YEL-AVD and three YEL-AND), and in Lindsey et al's study ${ }^{25}$ there were 14 SAEs (two YEL-AVD and three YEL-AND). In those aged $\geq 70$ years in Khromava et al's study, ${ }^{24}$ there were seven SAEs (three YEL-AVD and one YEL-AND), while in Lindsey et al's study, ${ }^{25}$ seven SAEs (two YEL-AVD and two YEL-AND) were observed. Monath et $\mathrm{al}^{27}$ extrapolated the 1,043,416 doses of vaccine given in the UK between 1995 and 1999 to be the same age structure as the much smaller UK General Practice Database. For example, for the age group $>75$ years he extrapolated that 8,595 YF vaccinations were given to that entire age group from the 104 doses recorded in the UK General Practice Database. ${ }^{27}$

Since this systematic review was published, four further studies of at-risk groups were published. A Cochrane review of 484 patients with HIV infection identified no SAEs after YF vaccination. ${ }^{28}$ A study of 19 solid organ transplant recipients who accidentally received YFV 3-241 months after transplantation while taking immunosuppressive medications identified no YF-AND or YF-AND cases. ${ }^{29}$ An analysis of 65 YEL-AVD cases in the CDC database as of January 2011 (three were deleted as age and sex were unknown) and two cases from subsequently published articles (no search strategy listed) for a total of 64 cases. Seligman ${ }^{30}$ noted his listing was "modified by an analysis of articles cited in a comprehensive review" by Thomas et a ${ }^{10}$ but did not add any further cases. He noted "Although the definition of viscerotropic disease has been revised by the Brighton Viscerotropic Working Group, in many instances insufficient data are available to evaluate a given case. Accordingly acceptance by CDC was the primary criterion for inclusion”. He noted, denominator data are not available for these cases but argued (inappropriately) "Fortunately, the prevalence of the risk factor in the population can be substituted for the denominator in calculations of odds ratios". Important findings are that of the 29 cases of YEL-AVD he identified who were $\geq 56$ years, of which 26 were male and three were female, and there were 15 cases in women aged 19-34 years. He did not provide age- and sex-specific denominators for all individuals.

Rafferty et al ${ }^{31}$ updated Thomas et al's ${ }^{12}$ literature search, included five additional articles and assessed studies with the updated Brighton Collaboration YEL-AVD criteria published in 2012. They identified the same problems in the five pharmacovigilance databases. They recalculated relative risk reduction (RRRs) for individual studies: "The re-calculated RRRs for Martin et $\mathrm{al}^{26}$ and Khromava et $\mathrm{al}^{24}$ comparing elders ( $\geq 65$ and $\geq 60$ respectively) to the reference population (15-64 and $<60$ respectively) stayed high, and were significant in three of four re-calculations". Their recomputed relative risks for Lindsey et $\mathrm{al}^{25}$ were unchanged compared to the original, and were nonsignificant. They found no cases in people $\geq 65$ years in the Australian databases (Lawrence et $\mathrm{al}^{32}$ ), the recalculated result for Martin et $\mathrm{al}^{26}$ was nonsignificant, and noted Monath et $\mathrm{al}^{27}$ did not provide sufficient information. The abstract concluded: "the evidence remains limited" and the article concluded: "In summary, our findings suggest there may be an increased risk of YEL-AVD among unexposed elders, although the overall risk remains low".

\section{Conclusion for the safety and immunogenicity of the vaccine}

The systematic reviews assessed here identify that the general population is at very low risk for SAEs from any of the current YF vaccines (except that there are no published data for the Chinese Tiantan vaccine). Published studies identify immunosuppressed individuals, those with a thymectomy or thymic disease or thymic radiation, children $\leq 6$ months, and those with severe egg allergy as at risk, and it is contraindicated for these groups.

A systematic review of studies of how long immunity lasts after vaccination with yellow fever vaccine identified eight studies between 1947 and 2012. ${ }^{33}$ Of the six studies of 17D (vaccine type not specified), the four with the longest interval identified $80.6 \%$ neutralizing antibodies in 149 military personnel after $>30$ years, $100 \%$ neutralizing antibodies in 24 military personnel after $16-19$ years, 95\% neutralizing 
antibodies in elderly persons after a median of 14 years, and $74.5 \%$ neutralizing antibodies in 209 healthy Berlin residents after 10 years. This provides support for the need for only one YF vaccination (unless the vaccination did not provide a strong neutralizing antibody level).

\section{Conclusion and recommendations: implications for prescription and use}

The WHO article summarizing current evidence for all YF vaccines concludes that the vaccine can be offered to HIV+ individuals with CD4 T-cell counts $\geq 200$ cells $/ \mathrm{mm}^{3}$, that the benefits to pregnant women in endemic areas far outweigh the risk of transmission to the fetus or infant, it can be administered to children aged 6-8 months during epidemics when the risk of infection is high, and given with caution to those aged $\geq 60$ years. It should not be given to children $\leq 6$ months of age, those with severe egg allergy, or severe immunodeficiency. The article concluded that "protection appears to last at least 20-35 years and probably for life" and recommended "A single dose of YF vaccine is sufficient to confer sustained life-long protective immunity against YF disease; a booster dose is not necessary".

Research is currently focusing on production of an inactivated YF vaccine based on inactivation by $\beta$-propiolactone, ${ }^{34}$ hydrostatic pressure, ${ }^{35}$ or by using a nonreplicating vaccinia virus transfected with the gene encoding the precursor of the membrane and envelope of the 17D YFV. ${ }^{36}$

\section{Acknowledgments}

In 2010, the Global Advisory Committee on Vaccine Safety (GACVS) requested that the World Health Organization (WHO) commission an independent systematic review of the safety of YF vaccine. A systematic review was prepared for the WHO and GACVS by a research team at the University of Calgary headed by Professor Roger E Thomas. The focal contact person for the WHO was Dr Alejandro Costa with Dr Rosamund Lewis. There was extensive correspondence with the WHO focal person and Dr Rosamund Lewis, with additional correspondence with Dr Sergio Yactayo. The literature search for the current article is mainly based on the literature search for the commissioned systematic review, and updated. The initial literature search and systematic review was funded by The Global Alliance for Vaccines and Immunization (GAVI).

\section{Disclosure}

The scientific independence of the researchers was at all times maintained. The sponsors did not participate in the collection, analysis, or interpretation of data or the writing of the report. The author reports no conflicts of interest in this work.

\section{References}

1. World Health Organization. Vaccines and vaccination against yellow fever WHO Position Paper - June 2013. Epidemiol Rec. 2013;88(27):269-284. Available from: http://www.who.int/wer/en. Accessed March 23, 2015.

2. Barrett ADT, Teuwen DE. Yellow fever vaccine - how does it work and why do rare cases of serious adverse events take place? Curr Opin Immunol. 2009;21:308-313. doi:10.1016/j.coi.2009.05.018.

3. Stock NK, Boschetti N, Herzog C, Appelhans MS, Niedrig M. The phylogeny of yellow fever virus 17D vaccines. Vaccine. 2012;30:989-994 doi:10.1016/j.vaccine.2011.12.057.

4. Pfister M, Kürsteiner O, Hilfiker H, Favre D, Durrer P, Ennaji A, et al. Immunogenicity and safety of Berna-YF compared with two other 17D Yellow Fever vaccines in a phase 3 clinical trial. Am J Trop Med Hyg. 2005;72(3):339-346.

5. Akondy RS, Monson ND, Miller JD, et al. The yellow fever virus vaccine induces a broad and polyfunctional human memory CD8+ T cell response. J Immunol. 2009;183(12):7919-7930. doi:10.4049/ jimmunol.0803903.

6. Blom K, Braun M, Ivarsson MA, et al. Temporal dynamics of the primary human $\mathrm{t}$ cell response to yellow fever virus $17 \mathrm{D}$ as it matures from an effector- to a memory-type response. J Immunol. 2013;190:21502158. doi:10.4049/jimmunol.1202234.

7. Roukens AH, Soonawala D, Joosten SA, et al. Elderly subjects have a delayed antibody response and prolonged viraemia following yellow fever vaccination: a prospective controlled cohort study. PLoS One 2011;6(12):e27753. doi:10.1371/journal.pone.0027753.

8. World Health Organization Regional Office for the Western Pacific Region. Immunization Safety Surveillance: Guidelines for Immunization Programme Managers on Surveillance of Adverse Events Following Immunization. 2nd ed. Manila, Philippines: WHO Press; 2013.

9. Thomas RE, Spragins W, Lorenzetti DL. How many published cases of serious adverse events after yellow fever vaccination meet Brighton Collaboration diagnostic criteria? Vaccine. 2013;31:6201-6209.

10. Thomas RE, Lorenzetti DL, Spragins W, Jackson D, Williamson T. Active and passive surveillance of yellow fever vaccine 17D or 17DD-associated serious adverse events: Systematic review. Vaccine. 2011;29(28):4544-4555.

11. Thomas RE, Lorenzetti DL, Spragins W, Jackson D, Williamson T. Reporting rates of yellow fever vaccine 17D or 17DD-associated serious adverse events in pharmacovigilance data bases: systematic review. Curr Drug Saf. 2011;6(3):145-154.

12. Thomas RE, Lorenzetti DL, Spragins W, Jackson D, Williamson $\mathrm{T}$. The safety of yellow fever vaccine 17D or 17DD in children, pregnant women, HIV+ individuals, and older persons: systematic review. J Trop Med Hyg. 2012;86(2):359-372. doi:10.4269/ ajtmh.2012.11-0525.

13. Gershman MD, Staples JE, Bentsi-Enchill AD, et al. Viscerotropic disease: case definition and guidelines for collection, analysis, and presentation of immunization safety data. Vaccine. 2012;30: 5038-5058.

14. Rüggeberg JU, Gold MS, Bayas J-M, et al. Anaphylaxis: case definition and guidelines for data collection, analysis, and presentation of immunization safety data. Vaccine. 2007;25:5675-5684.

15. Sejvar JJ, Kohl KS, Gidudu J, et al. Guillain-Barré syndrome and Fisher syndrome: case definitions and guidelines for collection, analysis, and presentation of immunization safety data. Vaccine. 2011;29: 599-612.

16. Sejvar JJ, Kohl KS, Bilynsky R, et al. Encephalitis, myelitis, and acute disseminated encephalomyelitis (ADEM): case definitions and guidelines for collection, analysis, and presentation of immunization safety data. Vaccine. 2007;25:5771-5792. 
17. O’Conghaile SO, Alsharbaty MJ, McDermott SR, Conlon PJ, Royston MD, Dwyer RC. Fulminant hepatitis and multisystem organ failure following yellow fever vaccination: description of a fatal case. Anaesth Intensive Care. 2014;42(3):423-424.

18. Pires-Marczewski FC, Martinez VP, Nemirovsky C, Padula PJ. Intrathecal antibody production in two cases of yellow fever associated neurotropic disease in Argentina. J Med. 2011;83:2208-2212.

19. Breugelmans JG, Lewis RF, Agbenu E, et al. Adverse events following yellow fever preventive vaccination campaigns in eight African countries from 2007 to 2010. Vaccine. 2013;31:1819-1829.

20. Cottin P, Niedrig M, Domingo C. Safety profile of the yellow fever vaccine Stamaril $^{\mathrm{TM}}$ : a 17-year review. Expert Rev Vaccines. 2013;12(11): 1351-1368. doi:10.1586/14760584.2013.836320.

21. de Menezes Martins R, de Lourdes de S Maia M, dos Santos EM, Cruz RL de S, dos Santos PRG, Carvalho SMD, et al. Yellow Fever Vaccine Post-marketing Surveillance in Brazil. Procedia Vaccinol. 2010;2:178-183. doi:10.1016/j.provac.2010.07.012.

22. Barban V, Girerd Y, Aguirre M, et al. High stability of yellow fever 17D204 vaccine: a 12-year retrospective analysis of large-scale production. Vaccine. 2007;25(15):2941-2950.

23. Moulin J-C, Silvano J, Barban V, Riou P, Allain C. Yellow fever vaccine: comparison of the neurovirulence of new 17D-204 Stamaril ${ }^{\mathrm{TM}}$ seed lots and RK 168-73 strain. Biologicals. 2013;41:238-246. Available from: http://dx.doi.org/10.1016/j.biologicals.2013.04.005. Accessed March 23, 2015.

24. Khromava AY, Eidex RB, Weld LH, et al; The Yellow Fever Vaccine Safety Working Group. Yellow fever vaccine: an updated assessment of advanced age as a risk factor for serious adverse events. Vaccine. 2005;23:3256-3263.

25. Lindsey NP, Schroeder BA, Miller ER, et al. Adverse event reports following yellow fever vaccination. Vaccine. 2008;26:6077-6082.

26. Martin M, Weld LH, Tsai TF, et al; GeoSentinel Yellow Fever Working Group. Advanced age a risk factor for illness temporally associated with yellow fever vaccination. Emerg Infect Dis. 2001;7: 945-951.
27. Monath TP, Cetron MS, McCarthy K, et al. Yellow fever 17D vaccine safety and immunogenicity in the elderly. Hum Vaccin. 2005;1: 207-214.

28. Barte H, Horvath TH, Rutherford GW. Yellow fever vaccine for patients with HIV infection. Cochrane Database Syst Rev. 2014;1:CD010929. doi:10.1002/14651858.CD010929.pub2.

29. Azevedo L, Lasmar E, Contier F, et al. Yellow fever vaccination in organ transplanted patients: is it safe? A multicenter study. Transpl Infect Dis. 2012;14:237-241.

30. Seligman SJ. Risk groups for yellow fever vaccine-associated viscerotropic disease (YEL-AVD). Vaccine. 2014;32:5769-5775. doi:org/10.1016/j.vaccine.2014.08.051.

31. Rafferty E, Duclos P, Yactayo S, Schuster M. Risk of yellow fever vaccine-associated viscerotropic disease among the elderly: a systematic review. Vaccine. 2013;31:5798-5805. doi.org/10.1016/j.vaccine. 2013.09.030.

32. Lawrence GL, Burgess MA, Kass RB. Age-related risk of adverse events following yellow fever vaccination in Australia. Commun Dis Intell. 2004;28:244-248.

33. Gotuzzo E, Yactayo S, Córdova E. Efficacy and duration of immunity after yellow fever vaccination: systematic review on the need for a booster every 10 years. Am J Trop Med Hyg. 2013;89(3):434-44. doi: 10.4269/ajtmh.13-0264. Accessed April 8, 2015.

34. Monath T, Fowler E, Johnson C, et al. An inactivated cell-culture vaccine against yellow fever. $N$ Engl J Med. 2011;364:1326-1333.

35. Gaspar L, Mendes Y, Yamamura A, et al. Pressure inactivated yellow fever 17DD virus: implications for vaccine development. J Virol Methods. 2008;150:57-62.

36. Schafer B, Holzer G, Joachimsthaler A, et al. Preclinical efficacy and safety of experimental vaccines based on non-replicating vaccinia vectors against yellow fever. PLoS One. 2011;6(9):e24505.
Vaccine: Development and Therapy

\section{Publish your work in this journal}

Vaccine: Development and Therapy is an international, peer-reviewed, open access journal that spans the spectrum of vaccine design and development through to clinical applications. The journal is characterized by the rapid reporting of application notes, reviews, original research and clinical studies in all therapeutic areas. Clinical outcomes, patient safety,

\section{Dovepress}

and programs for the development and effective, safe, and sustained use of vaccines will be a feature of the journal. The manuscript management system is completely online and includes a very quick and fair peer-review system. Visit http://www.dovepress.com/testimonials.php to read real quotes from published authors. 